http://dx.doi.org/10.35381/r.k.v5i4.938

\title{
Provisiones por desahucio y jubilación patronal su reconocimiento y medición contable y tributario
}

\section{Provisions for eviction and employer retirement, their recognition and accounting and tax measurement}

\author{
Marco Antonio Chacón-González \\ marco.chacon08@est.ucacue.edu.ec \\ Universidad Católica de Cuenca, Cuenca \\ Ecuador \\ https://orcid.org/0000-0002-6047-1256 \\ Verónica Paulina Moreno-Narváez \\ veronica.moreno@ucacue.edu.ec \\ Universidad Católica de Cuenca, Cuenca \\ Ecuador \\ https://orcid.org/0000-0003-1517-6124 \\ Jaime Fabián Díaz-Córdova \\ ifdiazc@psg.ucacue.edu.ec \\ Universidad Católica de Cuenca, Cuenca \\ Ecuador \\ https://orcid.org/0000-0002-5406-4160
}

Recibido: 01 de junio de 2020 Revisado: 20 de junio de 2020 Aprobado: 02 de septiembre de 2020 Publicado: 24 de septiembre de 2020 


\title{
RESUMEN
}

El objetivo del presente estudio es diseñar una guía metodológica para el tratamiento contable y tributario de las Provisiones por desahucio y jubilación patronal su reconocimiento y medición contable y tributario de beneficios laborales bajo NIIF para PYMES de la provincia del Azuay para la determinación adecuada de la base tributaria. tiene por carácter metodológico un tipo descriptivo. En Ecuador la normativa tributaria aplicada para las provisiones por desahucio y jubilación patronal han cambiado en reiteradas ocasiones, para los periodos fiscales 2018, 2019 y 2020 no son deducibles de impuesto a la renta. Los impuestos diferidos generados por las provisiones de jubilación patronal y desahucio se compensan en la declaración de impuesto a la renta a las sociedades en los periodos fiscales que la empresa haya cancelado estos beneficios, disminuyendo la base tributaria mediante la reversión de diferencias temporarias, reduciendo el valor a pagar por impuesto a la renta.

Descriptores: Tributación; impuesto sobre la renta; gasto; derecho laboral; gestión de personal. (Palabras tomadas del Tesauro UNESCO).

\begin{abstract}
The objective of this study is to design a methodological guide for the accounting and tax treatment of Provisions for eviction and employer retirement, their recognition and accounting and tax measurement of labor benefits under IFRS for SMEs of the province of Azuay for the adequate determination of the base tax. its methodological character is a descriptive type. In Ecuador, the tax regulations applied to provisions for eviction and employer retirement have changed repeatedly, for fiscal periods 2018, 2019 and 2020 they are not deductible from income tax. The deferred taxes generated by the provisions for employer retirement and eviction are offset in the corporate income tax return in the fiscal periods that the company has canceled these benefits, reducing the tax base by reversing temporary differences, reducing the value to pay for income tax.
\end{abstract}

Descriptors: Taxation; income tax; expenditure; labour law; personnel management. (Words taken from UNESCO Thesaurus). 


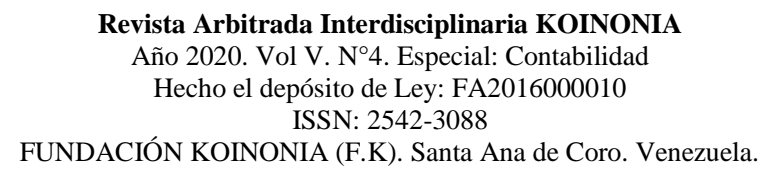

Marco Antonio Chacón-González; Verónica Paulina Moreno-Narváez; Jaime Fabián Díaz-Córdova

\section{INTRODUCCIÓN}

En la república del Ecuador las empresas deben constituir provisiones para el pago de beneficios a empleados, como el desahucio y jubilación patronal, a partir de suposiciones actuariales, que midan de forma técnica la obligación contraída probable y el registro del gasto en el ejercicio económico correspondiente, según (Rosas \& Castro, 2016), el cumplimiento de las obligaciones tributarias está sujeto al conocimiento sobre el tema, en consecuencia, desconocer de la deducibilidad en la constitución de provisiones, ocasiona el incumplimiento del registro, afectando los estados financieros de las empresas, al presentarlos de forma errónea.

Las empresas en Ecuador que según los datos del Instituto Nacional de Estadística y Censos (INEC) están conformadas por las micro, pequeñas y medianas empresas que abarcan el $99.53 \%$ del Directorio de Empresas y Establecimientos (DIEE), mientras que las grandes empresas conforman el 0.47\% (INEC, 2019). La Superintendencia de Compañías Valores y Seguros (SUPERCIAS) en su resolución N08.G.DSC.010 y sus reformas, establece la obligatoriedad de elaborar y presentar los estados financieros bajo Normas Internacionales de Información Financiera (NIIF), o NIIF para Pequeñas y Medianas Entidades (PYMES), en consecuencia, para la presentación de estados financieros, las empresas consideraran entre otras, la sección de la norma correspondiente a los beneficios a empleados, bajo este contexto, las empresas deben conocer las implicaciones y alcances de la norma.

El artículo 10 de la Ley de Régimen Tributario Interno (LRTI) establece los gastos deducibles para efectos de determinación de la base imponible de impuesto a la renta, para el 2017 el numeral 13 permitía la deducibilidad de las provisiones por jubilación y desahucio, siempre que, se realice un estudio actuarial efectuado por una empresa especializada, el mencionado numeral se modifica con la Ley Orgánica para la Reactivación de la Economía, Fortalecimiento de la Dolarización y Modernización de la Gestión Financiera (Asamblea Nacional del Ecuador, 2015), estableciendo deducción fiscal a los pagos por concepto de desahucio y pensiones jubilares, reforma que aplica 
para el periodo fiscal 2018, estableciendo la exclusión de la deducibilidad a la constitución de provisiones, sin embargo, se reconocerá en concordancia con las Normas Internacionales de Información Financiera (NIIF), un activo por impuesto diferido que se compensará cuando se efectúe el pago (Registro Oficial Suplemento 150 de 29-dic.-2017).

Por otra parte, la Ley Orgánica de Simplificación y Progresividad Tributaria, establece que serán deducibles para impuesto a la renta, las provisiones para atender el pago de desahucio y jubilación patronal siempre que sean formuladas por un actuario, la disposición transitoria mencionada en esta normativa, establece que el cambio entrará en vigencia a partir del año 2021, el cambio representa un mayor esfuerzo en el control de provisiones y manejo de los activos por impuestos diferidos generados en los años en que la provisión no es considerada deducible, al mismo tiempo si el empleado se desvincula de la entidad sin tener derecho a jubilación patronal o desahucio, se debe reversar los valores provisionados, generando un ingreso gravado o no objeto de renta, dependiendo del periodo en que se originó y si fue tratado como deducible o no deducible de impuesto a la renta, de la misma manera se reversa el activo por impuesto diferido generado en los periodos fiscales 2018, 2019, 2020 (Servicios de Rentas Internas del Ecuador, 2019).

Partiendo de los supuestos anteriores, conocer la normativa tributaria y contable, es un factor determinante para la correcta aplicación, por esto la importancia de analizar la influencia de la deducibilidad tributaria en la constitución de provisiones por jubilación patronal y establecer el procedimiento para aplicar lo que requiere la normativa tributaria, de acuerdo con periodos fiscales, para determinar la base imponible. 


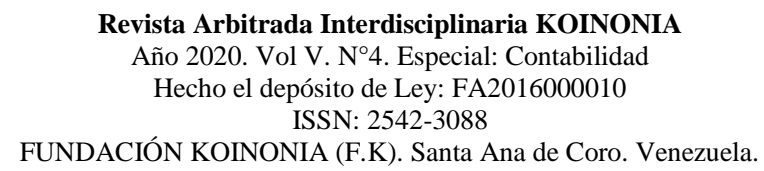

Marco Antonio Chacón-González; Verónica Paulina Moreno-Narváez; Jaime Fabián Díaz-Córdova

\section{Referencial teórico}

\section{Desahucio y jubilación patronal}

Conforme la legislación nacional el desahucio es la notificación por escrito, donde la persona trabajadora da a conocer al empleador su voluntad de dar por terminado el contrato laboral, el empleador tiene la obligación de bonificar con el $25 \%$ de la última remuneración por cada año laborado, en el plazo de 15 días posteriores a la notificación o aviso por parte del trabajador, incluso por medios electrónicos, cabe acotar si el trabajador no cumple un año de labores, no tiene derecho la bonificación por desahucio (Registro Oficial Suplemento 167 de 16-dic-2005).

Antes de la Ley Orgánica para la Justicia Laboral y Reconocimiento del Trabajo en el Hogar, el Código de trabajo permitía los contratos por tiempo definido o duración determinada, según (Lanas-Medina, 2015) los contratos tenían vigencia de un año pudiendo ser renovados por otro más, periodo en donde el empleador podía terminar la relación laboral mediante el desahucio que era solicitado ante un inspector de trabajo, solicitud que se realizaba por el trabajador o empleador, el inspector notificaba a las partes la terminación del contrato por desahucio.

Con la aplicación de la Ley Orgánica para la Justicia Laboral y Reconocimiento del Trabajo en el Hogar, se excluyen los contratos por tiempo definido y se elimina la participación del inspector de trabajo, con el segundo inciso del artículo 184 del Código de Trabajo, establece que todas las relaciones laborales terminadas en los casos establecidos por el artículo 169 del Código, numeral 2 tendrán derecho a la bonificación por desahucio, en otras palabras todo finiquito tiene derecho a desahucio, siempre y cuando el trabajador cumpla un año o más de labores.

En concordancia con el Código de Trabajo, la jubilación patronal es un beneficio que gozan los trabajadores que hayan laborado de forma continua o interrumpida con el mismo empleador por más de 25 años, también los trabajadores de 20 a 25 años que se desvinculan con despedido intempestivo gozarán de este beneficio de forma proporcional, para estos últimos el beneficio se pierde si llegasen a renunciar de forma 


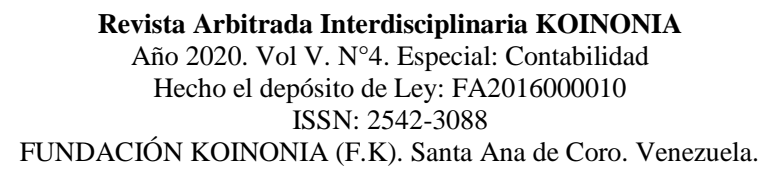

Marco Antonio Chacón-González; Verónica Paulina Moreno-Narváez; Jaime Fabián Díaz-Córdova

voluntaria, en cuanto al pago se establecen dos formas, con una pensión mensual vitalicia o con un fondo global (Registro Oficial Suplemento 167 de 16-dic-2005). Con el fin de garantizar el pago de un fondo global o pensión vitalicia el (Suplemento - Registro Oficial $N^{\circ}$ 732) expide el acuerdo ministerial MDT-2016-0099 y sus reformas, regulando la forma de cálculo, considerando las siguientes variables para la pensión mensual vitalicia: el fondo de reserva; las remuneraciones de los últimos cinco años; el tiempo de labor en la empresa, asimismo para el cálculo del fondo global se considera; el coeficiente de renta vitalicia; el valor de pensión mensual vitalicia; el valor de la décimo tercera y décimo cuarta remuneración.

Para (Pilalot \& Moreira, 2019) el pago de la jubilación patronal mediante el fondo global, da por terminada la relación laboral entre el empleador y trabajador, considerada una práctica que vulnera el derecho que debe ser vitalicio, la normativa permite las dos formas para el pago, si es mediante fondo global, es necesaria una solicitud del trabajador y será calculado en base al acuerdo ministerial antes mencionado.

Debido a que el cálculo es determinado por variables que necesitan ser medidas con fiabilidad es indispensable realizar un estudio actuarial, que mediante modelos estadísticos y matemáticos mida los posibles pasivos laborales de la empresa $u$ organización, que tendrá que hacer frente en el futuro. Es importante que las empresas conozcan de manera fiable el monto de sus obligaciones futuras por beneficios a empleados, para que no exista un impacto financiero, al realizar desembolsos por estos conceptos, para esto los actuarios determinan el valor presente de las posibles obligaciones, permitiendo cuantificar y constituir las provisiones por desahucio y jubilación patronal.

Los actuarios deben estar actualizados en NIIF, materia tributaria, Código de Trabajo, a partir de la implementación de las NIIF en Ecuador como técnica contable, nace la obligación de registrar los beneficios a empleados, mediante provisiones y adicional las leyes tributarias dictaminan si estas son deducibles de impuesto a la renta. El impuesto a las ganancias con la Norma Internacional de Contabilidad (NIC) 12 y la sección 29 de 


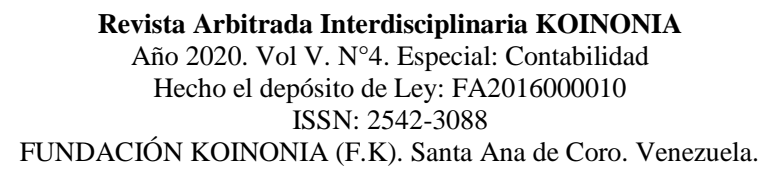

Marco Antonio Chacón-González; Verónica Paulina Moreno-Narváez; Jaime Fabián Díaz-Córdova

la NIIF PYMES deben ser considerados para el informe actuarial, debido a que, cuando se constituye una provisión no deducible se debe analizar si existe diferencia temporaria o permanente y constituir un activo por impuesto diferido para compensarse en otros periodos fiscales, estos activos deben ser revelados en los estados financieros de las empresas.

\section{Deducibilidad Tributaria}

Los sistemas impositivos que gravan renta, imponen su base a la riqueza que excede los costos y gastos incurridos para producirla, los países latinoamericanos, sin excepción, incorporan reglas de causalidad del gasto, para determinar la deducibilidad (Villagra-Cayamana \& Zuzunaga-del-Pino, 2014). En Ecuador la LRTI establece la restricción por causalidad especificando que, para determinar la base tributaria de impuesto a la renta se deducirá solo los gastos destinados a obtener, mantener o mejorar los ingresos de fuente ecuatoriana.

Para (Illescas-Hidalgo, 2017), la aplicación del principio de causalidad es un aspecto que queda al juicio del contribuyente y al final en potestad de la administración tributaria. Visto de esta forma las provisiones para jubilación patronal y desahucio, cumplen el principio de causalidad, dado que, sirven para los pagos de beneficios a empleados y el propósito de contrato de trabajadores es generar ingresos, sin embargo, para determinar la base imponible de impuesto a la renta, el numeral 13 del artículo 10 de la LRTI, tiene algunas reformas desde su creación en el año 2004, es así, que para analizar la deducibilidad se debe conocer la normativa vigente para cada periodo fiscal, como se observa en la tabla 1. 


\section{Tabla 1}

Gastos Deducibles de provisión por periodos fiscales

Periodo fiscal

\begin{tabular}{cl}
\hline \multirow{2}{*}{$2004-2007$} & $\begin{array}{l}\text { Son deducibles las provisiones para jubilación patronal, } \\
\text { formuladas por empresas especializadas en realizar informes }\end{array}$ \\
& $\begin{array}{l}\text { actuariales, aplica solo para personal que ha cumplido por lo } \\
\text { menos diez años consecutivos de trabajo en la misma empresa. } \\
\text { Son deducibles las provisiones por desahucio y jubilación }\end{array}$ \\
& $\begin{array}{l}\text { patronal, formuladas por empresas especializadas en realizar } \\
\text { informes actuariales, en el caso de jubilación patronal aplica solo } \\
\text { para personal que ha cumplido por lo menos diez años de trabajo } \\
\text { en la misma empresa. }\end{array}$ \\
& $\begin{array}{l}\text { No son deducibles las provisiones por desahucio o jubilación } \\
\text { patronal, los pagos efectuados si son deducibles, por lo que se }\end{array}$ \\
& $\begin{array}{l}\text { acepta un activo por impuesto diferido por este concepto. } \\
\text { Son deducibles las provisiones por desahucio y jubilación }\end{array}$ \\
& $\begin{array}{l}\text { patronal, formuladas por empresas especializadas en realizar } \\
\text { informes actuariales, para la provisión por jubilación patronal se }\end{array}$ \\
debe cumplir con las siguientes condiciones: \\
a. Personal que ha cumplido por lo menos diez años de trabajo \\
en la misma empresa. \\
b. Los aportes en efectivo sean administrados por empresas \\
especializadas administradoras de fondos.
\end{tabular}

Fuente: Suplemento del Registro Oficial No. 463 (17 de noviembre 2004).

Así mismo, es importante considerar que el Reglamento para la aplicación de la Ley de Régimen Tributario Interno (RLRTI) en el artículo 27, establece que son deducibles todos los costos y gastos necesarios, vinculados a la actividad económica de la empresa y devengados en el ejercicio económico, es decir se debe conocer en que periodo fiscal fue causado el gasto (Registro Oficial 448, 28-Febreo-2015). Para el caso de las provisiones por jubilación patronal y desahucio se debe establecer, en que año se causa la obligación, de esta manera si existe desvinculación laboral de un empleado con derecho a los beneficios antes mencionados y no se mantiene una provisión para el pago, se debe considerar el periodo en el que se causó el gasto, el valor proporcional del gasto que no se origina en el periodo corriente, debe tener el tratamiento de no 
deducible. Debido a que, los beneficios están en función del tiempo laborado y cada año requiere la estimación de pérdidas o ganancias actuariales por principio de devengo.

Por otra parte, si el empleado se desvincula de la empresa sin tener derecho a la jubilación patronal o desahucio y la empresa mantiene valores aprovisionados por estos conceptos se deben reversar, considerando que, si al constituirse el gasto fue deducible debe tratarse como ingreso gravado, en cambio si fue no deducible el ingreso es no objeto de impuesto a la renta, debido a que el gasto no fue utilizado para reducir la base imponible, el efecto se detalla en la figura 1.

Gasto deducible

Gasto no deducible
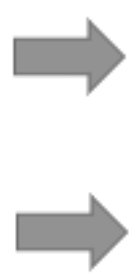

Ingreso gravado

Ingreso no objeto de renta

Figura 1: Afectación tributaria del reverso de provisión.

De este modo, es importante para las empresas llevar un control por empleado de las provisiones constituidas, para determinar de forma correcta la base imponible de impuesto a la renta. Por otro lado, el cumplimiento correcto de las obligaciones tributarias, está ligado al conocimiento de la normativa contable, según (Paredes-Floril, 2016) el $47,6 \%$ de las personas encuestadas en su estudio, no maneja un conocimiento de sus obligaciones y derechos, por cuanto, es importante la capacitación por parte de las entidades de control debido al constante cambio de la normativa tributaria. (Piedra, et al., 2016) indican que pocos empresarios participan en procesos de capacitación tributaria, evidenciado que existe poca preparación por parte de los contribuyentes.

La provisión para la atención de los beneficios a empleados por jubilación patronal y desahucio, constituye un gasto que al no ser registrado, distorsiona el resultado del 
ejercicio afectando a los estados financieros, (Ramírez, Ortiz, \& Tacuri, 2019) señalan que en su caso de estudio, la empresa no realiza provisión de beneficios por jubilación patronal o desahucio, debido a que la administración considera que realizar los pagos sin constituir provisiones no representa un impacto significativo en sus estados financieros, del mismo modo, en el caso de estudio de (Franco, Proaño, \& Salcedo, 2018) señalan que la empresa no realiza provisión por desahucio o jubilación patronal, sin embargo, ellos lo atribuyen este hecho al desconocimiento del personal para la aplicación de la norma. Por lo que se evidencia el desconocimiento de la norma en cuanto a la deducibilidad y su adecuada aplicación.

\section{Normas Internacionales de Información Financiera en el Ecuador}

Las NIIF en las últimas décadas se generalizaron como el modelo a seguir, con el propósito de tener un mayor grado de homogeneidad en la presentación de información financiera, donde su dinámica implica nuevas y mayores exigencias (Ruíz-Sánchez, 2017). Según (Salazar, 2013), se señalan como ventajas y desventajas de la implementación las siguientes: Ventajas.- mejora la calidad de información; separa la información tributaria de la financiera; ayuda para propósitos de información interna. Desventajas.- Incremento de costos administrativos, por la necesidad de capacitación; incremento de carga operativa; Riesgos de una mala implementación, debido a errores de interpretación o vacíos legales.

En Ecuador antes de la implementación de las NIIF, la normativa contable que aplicaban las empresas eran las Normas Ecuatorianas de Contabilidad (NEC). Según (Pérez-Rico, 2017) las NEC están basadas en las NIC, que son las primeras normas emitidas por el Consejo de Normas Internacionales de Contabilidad (IASB), el problema que tuvieron las NEC es que no se actualizaban al mismo ritmo que las NIC, lo que ocasionó un problema en la comparabilidad de los estados financieros de empresas ecuatorianas con las empresas a nivel mundial. La (Superintendencia de Compañías, 2008) mediante resolución $N^{\circ}$ 08.G.DSC.010 establece el cronograma de 


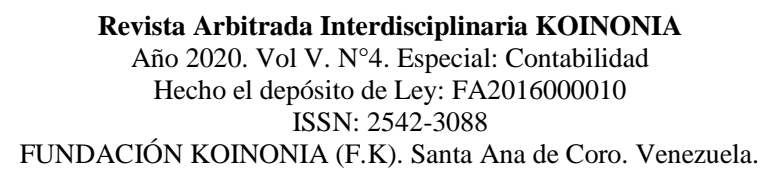

Marco Antonio Chacón-González; Verónica Paulina Moreno-Narváez; Jaime Fabián Díaz-Córdova

implementación de las NIIF en el Ecuador, estableciendo tres grupos: a partir del 2010 las compañías reguladas por la Ley de Mercado de Valores y empresas de auditoría externa; a partir del 2011 las compañías con activos superiores o iguales cuatro millones de dólares; para el 2012 las empresas con activos inferiores a cuatro millones y con ventas que no sobrepasen los cinco millones. Así mismo, la SUPERCIAS mediante resolución No. SCVS-INC-DNCDN-2019-0009 publicada en registro oficial No. 39 del 13 de septiembre de 2019, deja abierta la posibilidad, de que, si así lo requieren, las empresas apliquen NIIF para las PYMES, siempre que no se encuentren inscritas en el Catastro Público del Mercado de Valores, tomando en cuenta la normativa para la transición de NIIF completas a NIIF para las PYMES (Superintendencia de Compañías, 2019).

Según (Quizhpi, et al., 2019), las NIIF para las PYMES es aplicada en empresas en las que la información financiera es de interés solo para sus socios y no cotizan en la bolsa de valores, no obstante, en Ecuador hasta el año 2019 las empresas con activos mayores a cuatro millones de dólares, ventas mayores a cinco millones de dólares y de prestación de servicios de auditoría externa, estaban obligadas a implementar NIIF completas, a pesar de no cotizar en bolsa. Si bien la resolución No. SCVS-INC-DNCDN2019-0009 deja abierta la posibilidad a las empresas que no cotizan en bolsa de aplicar NIIF para las PYMES, para el cambio deben tener un periodo de transición contemplado en la normativa, pudiendo ser el periodo 2019 e implementarlo desde el 1 de enero de 2020, debido a esto existen numerosas empresas que conforme sus políticas contables, continúan implementando NIIF completas.

Las normas que establecen las bases para la presentación de estados financieros son; la NIC 1 y la sección 3 de las NIIF para PYMES, con el objetivo de asegurar de que los estados financieros sean comparables, tanto en la misma entidad al respecto de otros periodos, como con otras entidades (IASC Foundation Education, 2009). Según (Jiménez-Aguirre, 2015) las NIIF se emiten para proporcionar información comparativa, transparente y comprobable a participantes de los mercados capitales mundiales. Por 


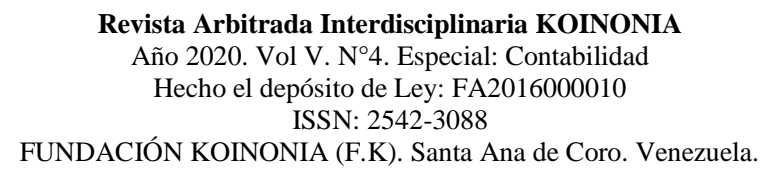

Marco Antonio Chacón-González; Verónica Paulina Moreno-Narváez; Jaime Fabián Díaz-Córdova

otra parte la NIC 1 establece el conjunto completo de estados financieros que se van a presentar los mismos que son: un estado de situación financiera al final del periodo, un estado del resultado y otro resultado integral del periodo, un estado de cambios en el patrimonio del periodo, un estado de flujos de efectivo del periodo, notas que incluyan un resumen de las políticas contables significativas y otra información explicativa.

El conjunto completo de estados financieros debe tener información comparativa, con respecto al periodo anterior, en todos los importes incluidos del periodo presentado, debiendo describir la información relevante para la compresión de los estados financieros del periodo corriente. Debido a esto las empresas deben tener una clasificación de las partidas contables de manera uniforme (IASB, 2015).

La jubilación patronal y el desahucio están regulados dentro de la NIC 19 y la Sección 28 de las NIIF para PYMES, como beneficios post empleo que se pagan después de terminar la relación laboral, estableciendo que se requieren suposiciones actuariales para el registro contable de las provisiones con la posibilidad de obtener ganancias 0 pérdidas actuariales, la NIC 19 en el párrafo 67 y la sección 28 en el párrafo 18 de las NIIF para las PYMES, en concordancia establecen que el método de valoración actuarial a utilizar es el de unidad de crédito proyectada, para determinar el valor presente de los beneficios, distribuyéndose entre los periodos de servicio (IASC Foundation Education, 2009) (IASB, 2015). En otras palabras, el gasto por provisión de beneficios laborales debe ser reconocido durante los periodos de servicio, necesitando de un estudio actuarial que determine el valor presente de las obligaciones, para registrar el gasto en el resultado del periodo y las ganancias o pérdidas actuariales que pertenecen a otros resultados integrales.

La norma que regula el impuesto a las ganancias más conocido como impuesto a la renta en Ecuador, es la NIC 12 de las NIIF completas y la Sección 29 de la NIIF para PYMES, conjugando la normativa contable con la tributaria, para el caso de las provisiones por jubilación patronal y desahucio, no son deducibles de impuesto a la renta para los periodos fiscales 2018, 2019 y 2020, sin embargo, el RLRTI en el 


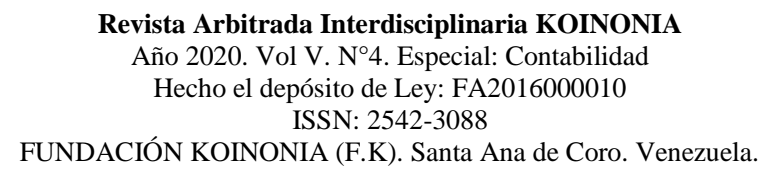

Marco Antonio Chacón-González; Verónica Paulina Moreno-Narváez; Jaime Fabián Díaz-Córdova

numeral 11 del artículo innumerado agregado a continuación del artículo 28 reconoce un impuesto por activo diferido, volviéndose el gasto por provisión una diferencia temporaria que se recupera en periodos futuros, cuando se realice el pago del beneficio al empleado. (Ruiz, et al., 2019) argumentan que las diferencias temporarias se generan de la diferencia entre la base fiscal y la base contable, debido a que la normativa tributaria no considera todos los gastos de la empresa deducibles de renta, sin embargo, serán recuperados en periodos posteriores.

El activo por impuesto diferido se debe reconocer considerando la tarifa de impuesto a la renta del periodo multiplicada por la diferencia temporaria, es decir, el valor de impuesto a la renta a recuperar, para (Cárdenas-Pañi, et al., 2020) el reconocimiento de un activo por impuesto diferido se debe considerar que su contrapartida afecta el estado de resultados, definiendo el valor del gasto por impuesto a la renta corriente.

En el caso del desahucio es más común en las empresas tengan la obligación de pagar el beneficio, debido a que se goza del beneficio a partir de un año de labor con el mismo empleador, por lo que, si pagó el beneficio en el año 2019 debió afectar el pago con la provisión, si la misma no fue suficiente registrar un gasto, al mismo tiempo reversar el activo por impuesto diferido para compensarlo en la declaración de impuesto a la renta del 2019 disminuyendo la base fiscal, debido a esto llevar un control por empleado, de la provisión y del activo por impuesto diferido, se vuelve imprescindible para el cumplimiento de la normativa fiscal y contable. De igual forma para el caso de la Jubilación patronal, siendo menos común, debido a que, se goza del beneficio a partir de laborar con el mismo empleador, durante más de 25 años con renuncia voluntaria y de 20 a 25 años con despido.

Por lo tanto, el objetivo del presente estudio es diseñar una guía metodológica para el tratamiento contable y tributario de las Provisiones por desahucio y jubilación patronal su reconocimiento y medición contable y tributario de beneficios laborales bajo NIIF para PYMES de la provincia del Azuay para la determinación adecuada de la base tributaria. 


\section{MÉTODO}

El presente estudio tiene por carácter metodológico un tipo descriptivo con diseño de campo no experimental. Los datos recolectados fueron de fuente primaria, mediante encuestas a los contadores de empresas de la provincia del Azuay obligadas a llevar contabilidad, para lo cual se realizó un muestreo por conveniencia y no probabilístico de 45 empresas, los aspectos principales a analizar fueron: constitución de provisiones por desahucio o jubilación patronal; conocimiento de la normativa tributaria y contable; aplicación correcta de la normativa; influencia de la deducibilidad de provisiones. Se empleó la estadística descriptiva para el análisis de la información recopilada.

\section{RESULTADOS}

La encuesta aplicada a contadores de diferentes empresas del Azuay, estaba conformada por 17 preguntas sobre la aplicación de gastos deducible, se determinó que la mayoría de encuestados (47\%) tiene de cero a tres años de experiencia en el cargo de contador, seguido de personas con más de 12 años de experiencia (33\%), en cuanto a la cantidad de empleados respecta, el 53\% de empresas encuestadas tienen de 1 a 15 empleados, mientras que, el $20 \%$ más de 60 , datos que sirven para caracterizar las empresas encuestadas.

Como se puede observar en la figura 2, apenas el $16 \%$ de los encuestados afirma tener un amplio conocimiento de la normativa tributaria y contable en la deducibilidad de las provisiones por jubilación patronal y desahucio, es así que se evidencia que entre los encuestados existe un alto porcentaje de contadores que no conocen por completo la normativa contable y tributaria aplicable. 


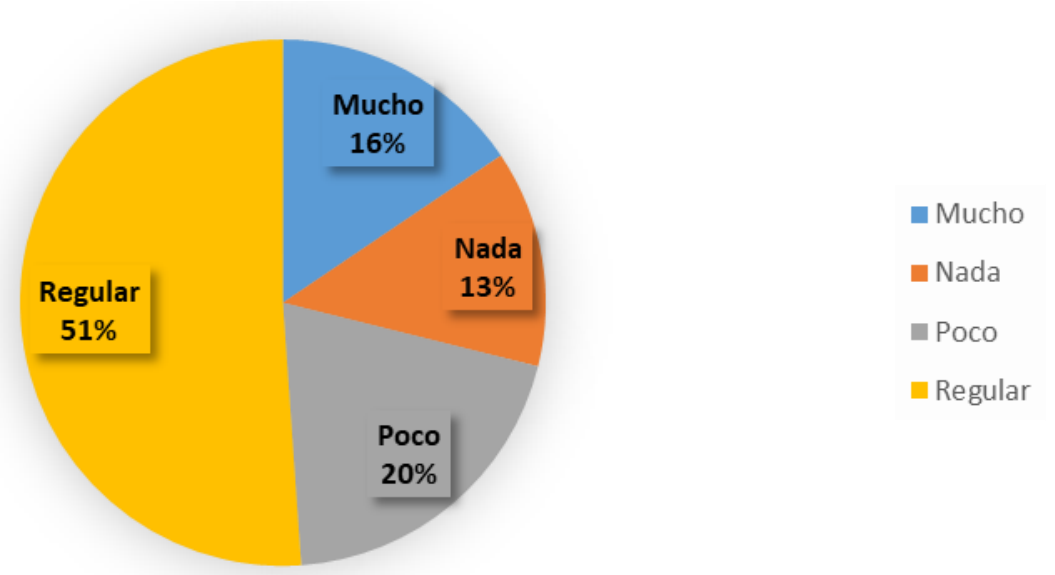

Figura 2. Conocimiento tributario y NIIF, en la provisiones de jubilación patronal y desahucio.

Fuente: Encuesta aplicada a los Contadores de empresas en el Azuay.

Del total de encuestados, el $60 \%$ afirmó no haber realizado un estudio actuarial en el periodo fiscal 2019, a pesar de ser una obligación para el cumplimiento de las NIIF completas o para PYMES, esto demuestra que un alto porcentaje de empresas no está aplicando de forma adecuada la normativa. En los periodos fiscales 2017 y anteriores, el $53 \%$ de las empresas ha constituido provisiones por desahucio y jubilación patronal, es importante acotar que en esos años la provisión era deducible si se contaba con un estudio actuarial, mientras que, para los periodos fiscales 2018 y 2019 se redujo al $47 \%$ es decir, la deducibilidad tributaria tiene cierta afectación en la decisión de constituir provisiones, dado que, para estos periodos fiscales, las empresas no deben deducir de su base fiscal el gasto por la provisión, generando una diferencia temporaria que será compensada al momento del pago de los beneficios laborales.

Como se destaca en la figura 3 , el $73 \%$ de encuestados no han realizado la generación de un activo por impuesto por jubilación patronal o desahucio, a pesar de que, el RLRTI permite la aplicación de un activo por impuesto diferido por este concepto, así mismo, se consultó si se realizó reversión de un activo por impuesto diferido, el $82 \%$ no lo ha realizado, evidenciando un alto porcentaje de empresas que no han aplicado activos 
por impuestos diferidos para reducir su base fiscal, a pesar de que el $53 \%$ de encuestados afirma tener desembolsos por pago de desahucio en el mismo periodo, mientras que en la pregunta acerca de la importancia del registro de provisiones el $66 \%$ indicó que es importante, contrastando con el resultado de la figura 3, por lo que se deduce, que el desconocimiento de la normativa contable y tributaria es la principal razón por la que no se generaron o reversaron activos por impuestos diferidos.

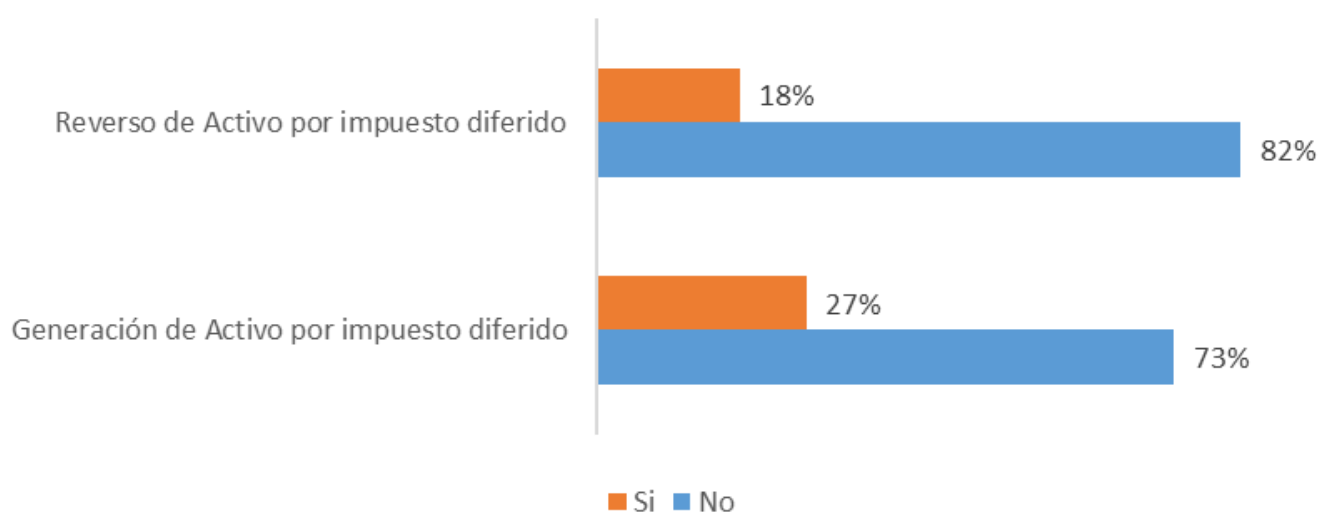

Figura 3. Reverso generación activo por impuesto diferido.

Fuente: Encuesta aplicada a los Contadores de empresas en el Azuay.

Como se expone en la figura 4, la mayoría de encuestados consideran, como la causa más común para no registrar provisiones por jubilación patronal y desahucio, el desconocimiento de la normativa tributaria y contable, seguido de las provisiones no son deducibles de impuesto a la renta en el periodo reportado y de solicitudes de la gerencia de no realizar la provisión. 


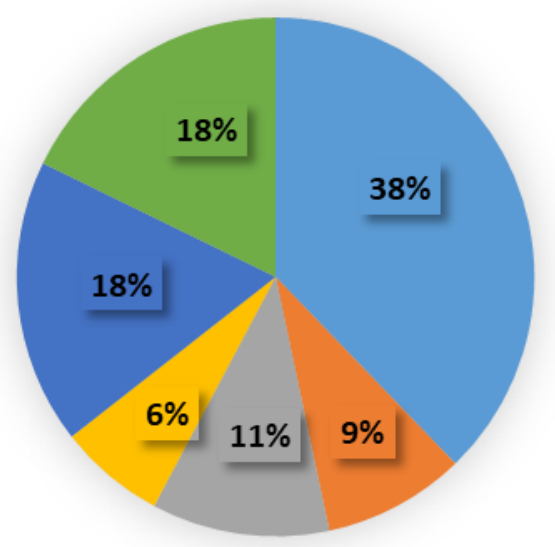

Desconocimiento de la normativa contable y tributaria.

- El gasto no es material para los estados financieros.

Estudio actuarial es muy costoso.

No son deducibles de impuesto a la renta la provisiones.

- No son deducibles de impuesto a la renta las provisiones.

- Por solicitud de gerencia.

Figura 4. Causas para no registrar provisiones por jubilación patronal y desahucio. Fuente: Encuesta aplicada a los Contadores de empresas en el Azuay

\section{PROPUESTA METODOLÓGICA DE TRATAMIENTO CONTABLE Y TRIBUTARIO}

De acuerdo con los resultados obtenidos, se considera conveniente diseñar una guía metodológica para el tratamiento contable y tributario de beneficios laborales bajo NIIF para las PYMES de la provincia del Azuay, que permita la correcta generación y reverso de activos por impuestos diferidos de las provisiones de desahucio y jubilación patronal, en cuatro etapas, comenzando por la determinación de las diferencias temporarias, hasta la liquidación tributaria del activo como se observa en la figura 5. Proporcionando pautas que ayuden a las PYMES en la provincia del Azuay a la adecuada determinación de la base tributaria de impuesto a la renta. 


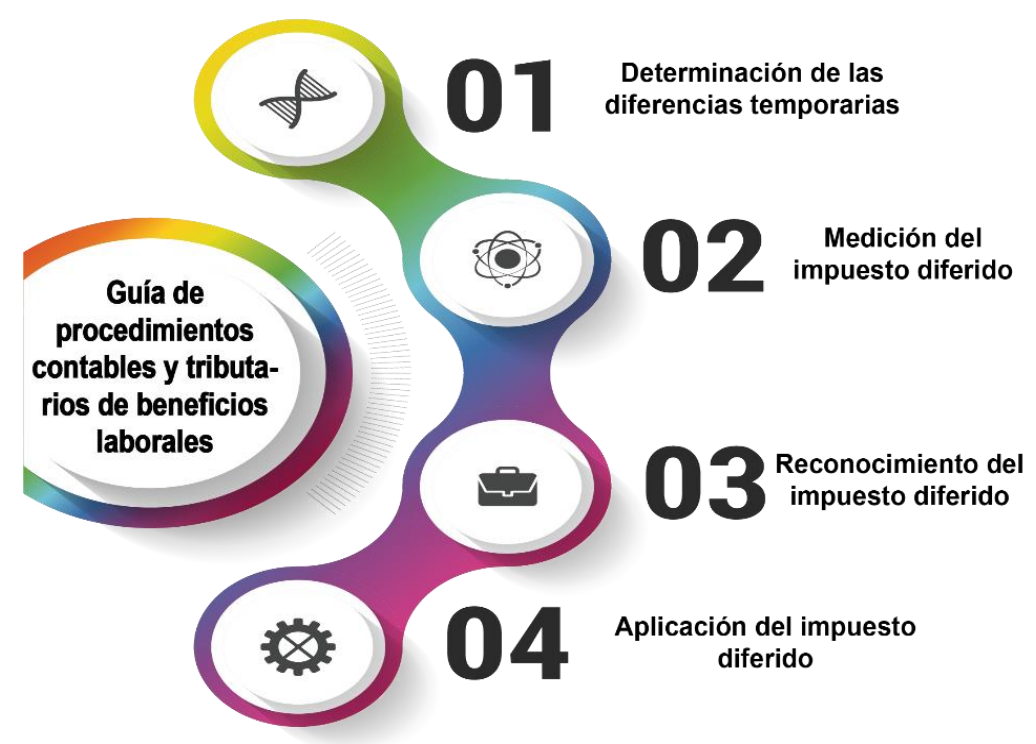

Figura 5. Metodológica para el tratamiento contable y tributario de beneficios laborales bajo NIIF para PYMES.

Fuente: Elaboración propia.

\section{Metodológica para el tratamiento contable y tributario de beneficios laborales bajo NIIF para las PYMES}

Objetivo: proporcionar a los contadores de las PYMES en la provincia del Azuay una herramienta que permita la adecuada medición y reconocimiento contable y tributario de los impuestos por activos diferidos, generados por las diferencias temporarias en la constitución de provisiones por desahucio y jubilación patronal.

Alcance: esta metodológica esta elaborada como una herramienta de apoyo para los contadores de las PYMES, especificando el tratamiento contable y tributario de los activos por impuesdos diferidos en la provisiones por desahucio y jubilación patronal en concordancia con la normativa tributaria Ecuatoriana.

Marco legal: el marco legal abarcado por la presente propuesta esta constituido por: NIIF para las PYMES sección 28 beneficios a los empleados; NIIF para las PYMES sección 29 impuesto a las gancias; Ley de Régimen Tributario Interno y su respectivo reglamento. 


\section{Determinación de diferencias temporarias}

Para determinar si existen diferencias temporarias deducibles bajo NIIF para las PYMES, la sección 29 párrafo 16 indica que se reconocen cuando a la empresa le resulta probable recuperar los importes por las diferencias fiscales, por lo que se debe analizar la normativa tributaria, estableciendo si el gasto registrado es no deducible de impuesto a la renta en el periodo corriente y es deducible en periodos fiscales futuros, estableciendo si la norma reconoce un activo por impuesto diferido, en el caso de las provisiones por jubilación patronal y desahucio se permite el reconocimiento. Las diferencias temporarias surgen cuando la base fiscal es mayor a la contable, debido a esto se debe analizar el tratamiento tributario según el periodo correspondiente, para establecer si existe diferencias temporarias, de acuerdo al RLRTI en el numeral 11 del artículo innumerado agregado a continuación del artículo 28 se reconoce un activo por impuesto diferido. El tratamiento de las diferencias temporarias por desahucio se indica en la tabla 2.

\section{Tabla 2}

Diferencias temporarias por desahucio según periodo fiscal

\begin{tabular}{ccc} 
Periodo fiscal & Tratamiento & Diferencia \\
\hline $2008-2017$ & Deducible & No hay diferencia fiscal \\
2019 & No deducible & Es diferencia temporaria \\
2020 & No deducible & Es diferencia temporaria \\
2021 en adelante & Deducible & No hay diferencia fiscal \\
\hline
\end{tabular}

Cabe recalcar que la provisión por jubilación patronal en los periodos fiscales entre 2008 y 2017, era deducible de impuesto a la renta si los empleados cumplían mínimo 10 años de labores, por lo contrario, la provisión por aquellos trabajadores con menos de 10 años tenía el tratamiento de no deducible, sin embargo, la normativa tributaria vigente en esos periodos no permitía el reconocimiento de activos por impuestos diferidos por este concepto, debido a esto no existen diferencias temporarias por provisión de jubilación patronal en aquellos periodos. 


\section{Medición del impuesto diferido}

De acuerdo con las NIIF para PYMES en la sección 29 párrafo 27, las empresas medirán el activo por impuesto diferido usando las tasas imponibles de impuesto a la renta según la legislación de cada país. En Ecuador la LRTI en el artículo 37 establece la tarifa de impuesto a la renta el $25 \%$ de la base imponible, si embargo, a la tarifa se deberá sumar tres puntos porcentuales en algunos casos como se puede observar en la tabla 3, asimismo en el segúndo artículo innumerado después del artículo 37.1 establece una rebaja de tres puntos porcentuales para las micro y pequeñas empresas.

\section{Tabla 3}

Tarifas de impuesto a la renta sociedades 2019

\section{Sociedades}

Sociedades en general Tarifa de impuesto

Micro y pequeñas empresas

$25 \%$

$22 \%$

Si no cumplen con informar el anexo accionistas, participes, socios, miembros del directorio y administradores (APS)

$28 \%$

Sociedades con socios o accionistas residentes en paraísos

fiscales o régimen fiscal de menor imposición

Fuente: Elaboración propia.

De igual manera se debe considerar que el párrafo 31 de la sección 29 de las NIIF para las PYMES establece que el activo por impuesto diferido debe someterse a revisión al final de cada periodo reportado y reversarse en el caso de que se estime que no posea suficiente ganancia fiscal para compensar el activo. El activo por impuesto diferido surge de la multiplicación de la tarifa de impuesto por la diferencia temporaria, que es el importe que se espera recuperar en periodos fiscales posteriores. 


\section{Reconocimiento del impuesto diferido}

Una vez establecida la diferencia temporaria y cuantificado el activo por impuesto diferido, se procede con el registro contable, como ejemplo la empresa "ABC" constituyó provisiones por desahucio y jubilación patronal según detalla la figura 5 .

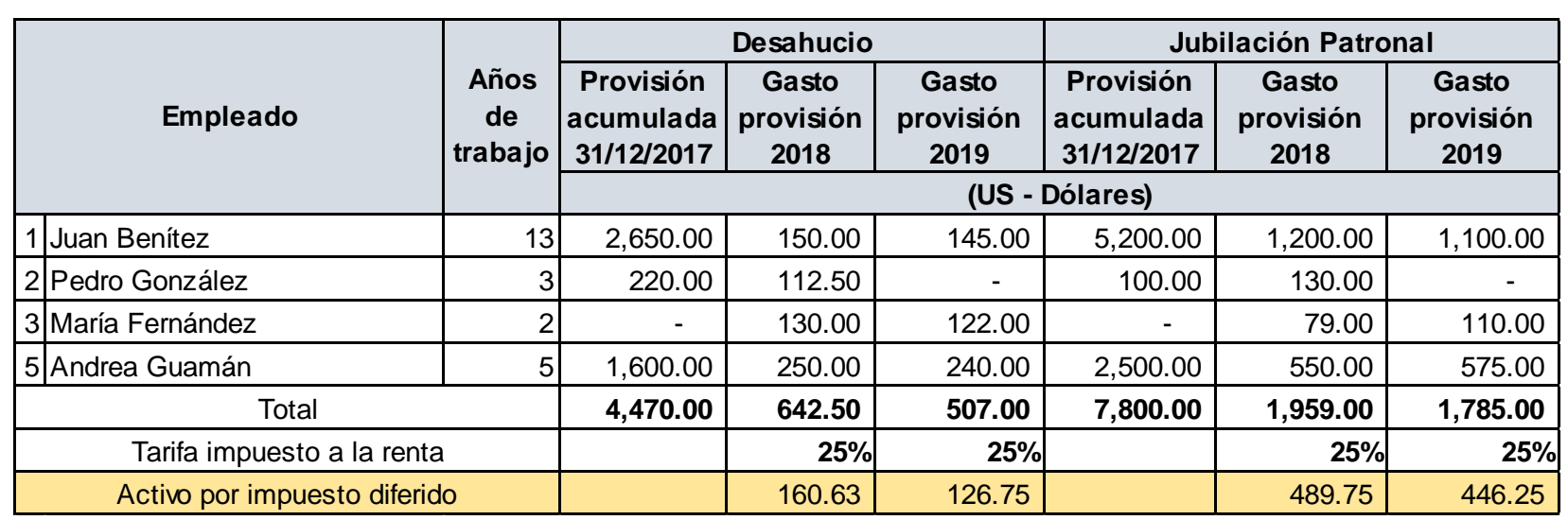

Figura 5. Ejemplo constitución de provisiones.

Se realiza el registro contable de la provisión del año 2019, asimismo, el activo por impuesto diferido considerando una tarifa de impuesto a la renta del $25 \%$, las diferencias temporarias son $\$ 507.00$ por desahucio y 1785.00 por jubilación patronal, por lo que multiplicado por la tarifa de renta quedaría un activo por impuesto diferido de $\$ 126.75$ y 446.25 como se detalla en la figura 6 . 
Revista Arbitrada Interdisciplinaria KOINONIA

Año 2020. Vol V. N². Especial: Contabilidad

Hecho el depósito de Ley: FA2016000010

ISSN: 2542-3088

FUNDACIÓN KOINONIA (F.K). Santa Ana de Coro. Venezuela.

Marco Antonio Chacón-González; Verónica Paulina Moreno-Narváez; Jaime Fabián Díaz-Córdova

\begin{tabular}{|c|c|c|c|}
\hline \multicolumn{4}{|c|}{ Año 2019} \\
\hline $\begin{array}{l}\text { Base NIIF } \\
\text { para las } \\
\text { PYMES }\end{array}$ & Concepto & Debe & Haber \\
\hline 28.15 & \multicolumn{3}{|c|}{$-\mathbf{X}-$} \\
\hline & Gasto provisión desahucio & 507.00 & - \\
\hline & Gasto provisión jubilación patronal & $1,785.00$ & - \\
\hline & Pasivo provisión desahucio & - & 507.00 \\
\hline & Pasivo provisión jubilación patronal & - & $1,785.00$ \\
\hline & \multicolumn{3}{|c|}{$\mathrm{P} / \mathrm{r}$ provisión desahucio y jubilación patronal según estudio actuarial } \\
\hline 29.27 & \multicolumn{3}{|c|}{$-\mathbf{X}-$} \\
\hline & Activo por impuesto diferido desahucio & 126.75 & - \\
\hline & Activo por impuesto diferido jubilación patronal & 446.25 & - \\
\hline & Gasto impuesto a la renta diferido & - & 573.00 \\
\hline & \multicolumn{3}{|c|}{$\mathrm{P} / \mathrm{r}$ reconocimiento de activo por impuesto diferido } \\
\hline
\end{tabular}

Figura 6. Contabilización impuesto diferido 2019.

A su vez, en el formulario de impuesto a la renta de sociedades se registra el valor neto des las diferencias temporarias, continuando con el ejemplo se suma las diferencias temporarias por provisión de desahucio y jubilación patronal para ser colocadas en el casillero 816 correspondiente a la generación, el gasto se registra en los casilleros 7056 y 7059 como detalla la figura 7 , notese que a pesar de ser un gasto no deducible en el periodo corriente no se registra valor en los casilleros 7057 y 7060 , debido a que la generación del activo por impuesto diferido en el casillero 816 ya incrementa la base imponible, por lo que si se adiciona como gasto no deducible tendría doble imposición. 


\begin{tabular}{|c|c|c|c|c|c|}
\hline GASTOS POR BENEFICIOS A LOS EMPLEADOS Y HONO & \multicolumn{2}{|c|}{ Gasto } & \multicolumn{3}{|c|}{ Valor no deducible } \\
\hline Jubilación patronal & 7056 & $1,785.00$ & 7057 & & \\
\hline Desahucio & 7059 & 507.00 & 7060 & & \\
\hline \multicolumn{6}{|c|}{ GENERACIÓN / REVERSIÓN DE DIFERENCIAS TEMPORARIAS (IMPUESTOS DIFERIDOS) } \\
\hline \multicolumn{3}{|c|}{ Por provisiones para desahucio pensiones jubilares patronales } & & 816 & $2,292.00$ \\
\hline
\end{tabular}

Figura 7. Registro generación diferencia temporaria formulario de renta sociedades. Fuente: Registro Oficial Suplemento 463 (17-nov.-2004).

\section{Aplicación del impuesto diferido}

Las entidades como establece el párrafo 37 de la sección 29 de la NIIF para la PYMES, compensaran los activos por impuestos diferidos si tienen el derecho legal exigible, la LRTI para los periodos fiscales 2018, 2019 y 2020 dispone que la provisión para jubilación patronal y desahucio no es deducible de impuesto a la renta, sin embargo, el gasto será deducible cuando el contribuyente se desprenda de recursos para cancelar los beneficios, a partir del 2021 por la disposición transitoria décima tercera de la Ley Orgánica de Simplificación y Progresividad Tributaria entra vigencia el artículo 13 numeral 3 disponiendo que los gastos por las provisiones antes referidas sean deducibles de impuesto a la renta, siempre que estén basadas en un estudio actuarial y para el caso de la jubilación patronal que el empleado cumpla mínimo 10 años de trabajo en la misma empresa.

EI RLRTI en el literal f del numeral 1 del artículo 28, establece que en la cancelación de los beneficios por jubilación patronal o desahucio se deben afectar las provisiones constituidas en años anteriores que hayan sido registradas como gasto deducible o no, las provisiones no utilizadas deben reversarse a resultados, como otros ingresos gravados o no objeto de impuesto a la renta en la proporción que fueron deducibles o no. Para el ejemplo se considera que el segundo empleado se desvincula de la empresa, recibiendo el beneficio de desahucio por $\$ 350.00$, asimismo, no cumple con los años de trabajo por jubilación patronal, debiendo reversar la provisión, con estas 
Revista Arbitrada Interdisciplinaria KOINONIA

Año 2020. Vol V. N². Especial: Contabilidad

Hecho el depósito de Ley: FA2016000010

ISSN: 2542-3088

FUNDACIÓN KOINONIA (F.K). Santa Ana de Coro. Venezuela.

Marco Antonio Chacón-González; Verónica Paulina Moreno-Narváez; Jaime Fabián Díaz-Córdova

premisas la contabilización se observa en la figura 8.

\begin{tabular}{|c|c|c|c|}
\hline \multicolumn{4}{|c|}{ Año 2019} \\
\hline $\begin{array}{c}\text { Base NIIF } \\
\text { para las } \\
\text { PYMES }\end{array}$ & Concepto & Debe & Haber \\
\hline \multirow[t]{5}{*}{28.21} & \multicolumn{3}{|l|}{$-X-$} \\
\hline & Pasivo provisión desahucio & 332.50 & - \\
\hline & Gasto por desahucio & 17.50 & - \\
\hline & Bancos & - & 350.00 \\
\hline & \multicolumn{3}{|c|}{$\mathrm{P} / \mathrm{r}$ pago de desahucio } \\
\hline \multirow[t]{5}{*}{ 28.21-28.24 } & \multicolumn{3}{|l|}{$-\mathrm{X}-$} \\
\hline & Pasivo provisión jubilación patronal & 230.00 & - \\
\hline & Otros ingresos reverso provisiones $\left({ }^{*}\right)$ & - & 100.00 \\
\hline & Otros ingresos reverso provisiones $\left({ }^{\star *}\right)$ & - & 130.00 \\
\hline & \multicolumn{3}{|c|}{$\mathrm{P} / \mathrm{r}$ reverso de provisión por jubilación patronal } \\
\hline \multirow[t]{4}{*}{29.37} & \multicolumn{3}{|l|}{$-\mathrm{X}-$} \\
\hline & Gasto impuesto a la renta diferido $(112.50 * 25 \%)$ & 28.13 & - \\
\hline & Activo por impuesto diferido desahucio & - & 28.13 \\
\hline & \multicolumn{3}{|c|}{$\mathrm{P} / \mathrm{r}$ aplicación del activo por impuesto diferido desahucio } \\
\hline 29.37 & \multicolumn{3}{|l|}{$-\mathbf{X}-$} \\
\hline & Gasto impuesto a la renta diferido $(130 * 25 \%)$ & 32.50 & - \\
\hline & Activo por impuesto diferido desahucio & - & 32.50 \\
\hline & $\mathrm{P} / \mathrm{r}$ reversión del activo por impue & $\mathrm{n}$ patronal & \\
\hline
\end{tabular}

$\left(^{\star}\right) \quad$ Ingreso no objeto de renta, para el periodo 2017 fue provisionado como gasto no deducible debido a que el empleado no tiene 10 años o más en la empresa.

$\left({ }^{\star *}\right)$ Ingreso no objeto de renta, para el periodo 2018 las provisiones son gastos no deducibles.

Figura 8. Contabilización aplicación y reversión activo por impuesto diferido.

En el formulario de impuesto a la renta para sociedades se debe registrar en el casillero 6077 el valor de la provisión no utilizada, para el ejemplo se reversa la provisión por jubilación patronal, ya que no cumplió con los años de labor requeridos para el beneficio, en el casillero 6078 se debe registrar si en inicio la provisión fue no deducible de impuesto a la renta, en este caso la provisión que mantenía el empleado es de $\$ 230.00$, el empleado tenía menos de 10 años de labores en la empresa, por lo que la provisión de jubilación patronal constituida hasta el 31-12-2017 debió ser tratada como no deducible, en cambio para el periodo 2018 no se permite la deducibilidad de la provisión independiente de los años de labor en la empresa, la asignación de los casilleros queda como se observa en la figura 9. 


\begin{tabular}{|c|c|c|c|c|c|}
\hline $\begin{array}{l}\text { GANANCIAS NETAS POR REVERSIONES DE PASIVOS POR BENEFICIOS A LOS } \\
\text { EMPLEADOS }\end{array}$ & \multicolumn{2}{|c|}{ Total ingresos } & \multicolumn{3}{|c|}{ Valor exento / no objeto } \\
\hline Jubilación patronal y desahucio & 6077 & 230.00 & & & 230.00 \\
\hline \multicolumn{4}{|c|}{ GENERACIÓN / REVERSIÓN DE DIFERENCIAS TEMPORARIAS (IMPUESTOS DIFERIDOS) } & \multicolumn{2}{|c|}{ Reversión } \\
\hline \multicolumn{3}{|c|}{ Por provisiones para desahucio pensiones jubilares patronales } & & 817 & 112.50 \\
\hline
\end{tabular}

Figura 9. Registro formulario renta sociedades generación diferencia temporaria.

Por otra parte, para la conciliación tributaria en el ejemplo se establece una utilidad de $\$ 15,000.00$, a la cual se debe restar la participación a trabajadores, las rentas exentas por las cuales no se generaron inpuestos diferidos y sumar los ingresos gravados generados por la reversión de provisiones constituidas como deducibles, obteniendo la base imponible corriente que sumado la generación y restando el reverso se determina la base fiscal. Asimismo, en el fomulario de impuesto a renta en el casillero 805 se coloca el valor neto del ingreso no objeto de impuesto a la renta por reverso de provisiones, con la tarifa impositiva del $25 \%$ el impuesto causado es $\$ 3674.88$, la conciliación tributaria y su efecto en la declaración de renta se observan en la figura 10. 


\section{Conciliación Tributaria 2019}

Utilidad del ejercicio

$15,000.00$

(-) Participación a trabajadores

$2,250.00$

(-) Otras rentas exentas e ingresos no objeto de Impuesto a la Renta

100.00

Subtotal

$12,650.00$

Generación activo por impuesto diferido en provisiones para desahucio pensiones

$(+)$ jubilares patronales $(1785+507)$

Reverso activo por impuesto diferido en provisiones para desahucio pensiones

$(-)$ jubilares patronales $(130+112.50)$

Base imponible

$14,699.50$

Impuesto a la renta corriente del ejercicio

$3,162.50$

Impuesto a la renta diferido

512.38

\begin{tabular}{|c|c|c|c|c|}
\hline \multicolumn{3}{|l|}{ UTILIDAD DEL EJERCICIO } & 801 & $15,000.00$ \\
\hline \multicolumn{3}{|l|}{ PÉRDIDA DEL EJERCICIO } & 802 & \\
\hline Base de cálculo de participación a trabajadores & & & 098 & $15,000.00$ \\
\hline \multicolumn{5}{|l|}{ DIFERENCIAS PERMANENTES } \\
\hline \multicolumn{3}{|l|}{ (-) Participación a trabajadores } & 803 & $2,250.00$ \\
\hline \multicolumn{3}{|l|}{ (-) Otras rentas exentas e ingresos no objeto de Impuesto a la Renta } & 805 & 230.00 \\
\hline GENERACIÓN / REVERSIÓN DE DIFERENCIAS TEMPORARIAS (IMPUESTOS DIFERIDOS) & \multicolumn{2}{|c|}{ Generación } & \multicolumn{2}{|c|}{ Reversión } \\
\hline Por provisiones para desahucio pensiones jubilares patronales & 816 & $2,292.00$ & 817 & 112.50 \\
\hline \multicolumn{3}{|l|}{ Utilidad gravable } & 836 & $14,699.50$ \\
\hline \multicolumn{3}{|l|}{ Impuesto a la renta a pagar } & 869 & 3674.88 \\
\hline
\end{tabular}

Figura 10. Conciliación tributaria y contable

\section{CONCLUSIONES}

Es importante constituir provisiones para reflejar el gasto en el periodo que corresponde, la NIIF para PYMES establece que el gasto por beneficios post-empleo debe ser distribuido entre los periodos laborados, precautelando que las empresas tengan recursos necesarios al momento de cancelar las obligaciones por beneficios a empleados. Para el tratamiento de las provisiones es importante que las personas responsables estén capacitadas en normativa contable y tributaria para un adecuado control. 


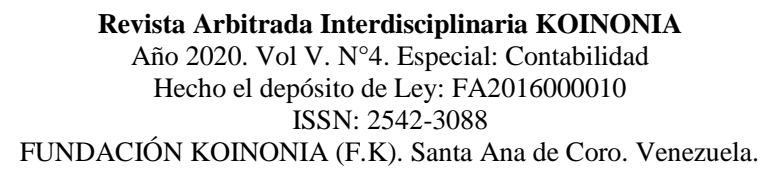

Marco Antonio Chacón-González; Verónica Paulina Moreno-Narváez; Jaime Fabián Díaz-Córdova

En Ecuador la normativa tributaria aplicada para las provisiones por desahucio y jubilación patronal han cambiado en reiteradas ocasiones, para los periodos fiscales 2018, 2019 y 2020 no son deducibles de impuesto a la renta, sin embargo, el fisco reconocerá la generación de un activo por impuesto diferido por este concepto, que será retribuido cuando la empresa se desprenda de recursos para cancelar la obligación, a partir del periodo fiscal 2021 son deducibles de impuesto a la renta siempre que las provisiones sean constituidas en base a un estudio actuarial y la jubilación patronal que el empleado tenga mínimo diez años de labores.

En el estudio evidencia que en la provincia del Azuay la mayoría de empresas no constituyen provisiones por jubilación patronal y desahucio, ni contratan a una empresa especializada para la realización de un estudio actuarial, sobre todo en las PYMES, asimismo, una de las razones para no constituir provisiones es que no son deducibles de impuesto a la renta, por lo que el gasto no puede ser considerado para la determinación de la base imponible tributaria en el periodo fiscal corriente.

Los contadores consideran que la principal causa para no constituir provisiones por beneficios a empleados, es el desconocimiento de la normativa, en cuanto a la aplicación de impuestos diferidos y su adecuada compensación tributaria, ya que en los años que no es deducible la provisión se deben reconocer activos por impuestos diferidos para ser retribuidos en periodos fiscales posteriores, en Ecuador se acepta la generación de un activo por impuesto diferido por provisiones de desahucio y jubilación patronal a partir del periodo fiscal 2018.

Para el registro contable del reconocimiento de los activos por impuestos diferidos, se debe considerar la tarifa de impuesto a la renta vigente por diferencias temporarias, en el formulario de impuesto a la renta para sociedades a pesar de que la provisión es un gasto no deducible, no se debe considerar como tal, debido a que el registro de la generación ya incrementa la base tributaria.

Los impuestos diferidos generados por las provisiones de jubilación patronal y desahucio se compensan en la declaración de impuesto a la renta a las sociedades en 
los periodos fiscales que la empresa haya cancelado estos beneficios, disminuyendo la base tributaria mediante la reversión de diferencias temporarias, reduciendo el valor a pagar por impuesto a la renta.

\section{FINANCIAMIENTO}

No monetario.

\section{AGRADECIMIENTO}

A los contadores de empresas de la provincia del Azuay; por el apoyo en el desarrollo de la investigación.

\section{REFERENCIAS CONSULTADAS}

Asamblea Nacional del Ecuador. (2015). Ley Orgánica para la Justicia Laboral y Reconocimiento del Trabajo en el Hogar. [Organic Law for Labor Justice and Recognition of Work at Home]. Recuperado de https://n9.cl/jknh

Cárdenas-Pañi, M., Narváez-Zurita, C., Erazo-Álvarez, J., \& Torres-Palacios, M. (2020). Conciliación de impuestos a las ganancias: Un estudio bajo la normativa fiscal y normativa contable [Reconciliation of income taxes: A study under tax regulations and accounting regulations]. Revista Arbitrada Interdisciplinaria Koinonía, 5(10), 757-773. http://dx.doi.org/10.35381/r.k.v5i10.715

Franco, J. C., Proaño, D. S., \& Salcedo, R. (2018). Provisiones por jubilación patronal y desahucio y su efecto en el impuesto a la renta [Provisions for employer retirement and eviction and its effect on income tax]. Observatorio de la Economía Latinoamericana. [Internet]. Recuperado de https://n9.cl/crh7g

IASB. (2015). NIIF para las PYMES. [IFRS for SMEs]. Recuperado de https://n9.cl/9xs5

IASC Foundation Education. (2009). NIC 19 Beneficios a los Empleados [IAS 19 Employee Benefits].

Illescas-Hidalgo, R. E. (2017). Costos y gastos deducibles en el impuesto sobre la renta: caso Nicaragua. [Income tax deductible costs and expenses: Nicaragua case]. Negotium, 13(39), 80-100. 
Instituto Nacional de Estadística y Censos. (2019). Directorio de empresas 2018 [Business Directory 2018]. Recuperado de https://n9.cl/y3z6j

International Accounting Standards Board (IASB). (2001). NIC 1 Presentación de Estados Financieros. [IAS 1 Presentation of Financial Statements]. Recuperado de https://n9.cl/hjru

Jiménez-Aguirre, R. (2015). Estándares Internacionales de Información Financiera: Algunos efectos en las organizaciones empresariales. [International Financial Reporting Standards: Some Effects on Business Organizations]. Lumina, 15, 112134. https://doi.org/10.30554/lumina.16.1671.2015

Lanas-Medina, E. (2017). Reformas al Código del Trabajo introducidas por la Ley de Justicia Laboral y Reconocimiento del Trabajo en el Hoga. [Reforms to the Labor Code introduced by the Law of Labor Justice and Recognition of Work at Home]. Foro, Revista De Derecho, (24), 5-22.

Paredes-Floril, P. R. (2016). Evasión tributaria vs. mecanismos de control implementados por la administración pública. [Tax evasion vs. control mechanisms implemented by the public administration]. RETOS Revista de Ciencias de la Administración y Economía, 6(12), 179-198.

Pérez-Rico, C., Méndez-Rojas, V., Fernández-García, C., Alvarado-Riquelme, M., \& Méndez-Rojas, P. (2017). Comunidad Andina de Naciones (CAN), Perú, Colombia, Bolivia y Ecuador: proceso de convergencia y adopción de las NIIF [Andean community of nations (CAN), Peru, Colombia, Bolivia and Ecuador: Process of convergence and adoption of ifrs]. Revista Economía $Y$ Política, 21(21), 65 - 74. https://doi.org/10.25097/rep.n21.2015.05

Piedra, A. F., Salinas, J. V., \& Vázquez, J. P. (2016). Caracterización tributaria en barrios urbanos populares: Caso parroquia El Vecino. [Tax characterization in popular urban neighborhoods: El Vecino parish case]. RETOS. Revista de Ciencias de la Administración y Economía, 6(11), 91-105. https://doi.org/10.17163/ret.n11.2016.06

Pilalot, L., \& Moreira, A. (2019). Efectos del pago del fondo global de jubilación patronal en el derecho de jubilación. [Effects of the payment of the global employer retirement fund on the right to retirement]. Revista Caribeña de Ciencias Sociales. [Internet]. Recuperado de https://n9.cl/4zi3 
Quizhpi, R. d., Narváez, C. I., \& Erazo, J. C. (2019). La gestión contable de las empresas comerciales en el marco de las NIIF para PY-MES [The accounting management of commercial companies within the framework of IFRS for SMEs]. Revista Arbitrada Interdisciplinaria KOINONIA, 2(4), 265-298. http://dx.doi.org/10.35381/r.k.v4i2.475

Ramírez, P., Ortiz, G., \& Tacuri, A. (2019). Provisiones para jubilación patronal y desahucio y su incidencia en los estados financieros. [Provisions for employer retirement and eviction and their impact on the financial statements]. Revista Observatorio de la Economía Latinoamericana. [Internet]. Recuperado de https://n9.cl/26iav

Registro Oficial 448, 28-Febreo-2015. Reglamento para la aplicación de la Ley de Régimen Tributario Interno. [Regulation for the application of the Internal Tax Regime Law]. Quito - Ecuador.

Registro Oficial Suplemento 150 de 29-dic.-2017. Ley Orgánica para la Reactivación de la Economía, Fortalecimiento de la Dolarización y Modernización de la Gestión Financiera. [Organic Law for the Reactivation of the Economy, Strengthening of Dollarization and Modernization of Financial Management]. Recuperado de https://n9.cl/xtomt

Registro Oficial Suplemento 167 de 16-dic-2005. Código del Trabajo. [Work code]. Recuperado de https://n9.cl/z3p5n

Registro Oficial Suplemento 463 de 17-nov.-2004. Ley De Régimen Tributario Interno LRTI. [Internal Tax Regime Law]. Recuperado de https://n9.cl/4i53

Rosas, C. E., \& Castro, O. F. (2016). Programa para incrementar la conciencia tributaria. [Program to increase tax awareness]. Revista de Investigación y Cultura, 5(2); $1-4$.

Ruiz, A. J., Narváez, C. I., \& Erazo, J. C. (2019). Tratamiento del impuesto diferido a partir de la valuación de activos biológicos bajo NIIF en la industria camaronera del Ecuador. [Treatment of the deferred tax from the valuation of biological activies low NIIF in the camaronera industry of Ecuador]. Revista Arbitrada Interdisciplinaria KOINONIA, 2(4), 299-320. http://dx.doi.org/10.35381/r.k.v4i2.476

Ruíz, M. d. (2017). Preparación en normas internacionales de información financiera en las Pymes de Villavicencio - Colombia Tendencias, 18(2), 27-44. http://dx.doi.org/10.22267/rtend.171802.74 
Ruíz-Sánchez, M. (2017). Preparación en normas internacionales de información financiera en las Pymes de Villavicencio - Colombia. [Preparation of international financial reporting standards for SMEs in Villavicencio - Colombia]. Tendencias, 18(2), 27-44. https://doi.org/10.22267/rtend.171802.74

Salazar, É. E. (2013). Efectos de la implementación de la NIIF para las PYMES en una mediana empresa ubicada en la ciudad de Bogotá. [Effects of the implementation of the IFRS for SMEs in a medium-sized company located in the city of Bogotá]. Cuadernos de Contabilidad, 14(35), 395-414.

Servicios de Rentas Internas del Ecuador. (2019). Ley Orgánica de Simplificación y Progresividad Tributaria. [Organic Law of Tax Simplification and Progressivity]. Recuperado de https://n9.cl/jcq36

Superintendencia de Compañías. (2008). Resolución No. 08.G.DSC. [Resolution No. 08.G.DSC]. Quito, Ecuador. Recuperado de https://n9.cl/br17

Superintendencia de Compañías. (2019). Resolución SCVS-INC-DNCDN-2019-0009. [Resolution SCVS-INC-DNCDN-2019-0009]. Quito, Ecuador: Registro Oficial No. 39. Recuperado de https://n9.cl/9u3p7

Suplemento - Registro Oficial № 732. Acuerdo ministerial MDT-2016-0099. [Ministerial agreement MDT-2016-0099]. Recuperado de https://n9.cl/wiqd8

Suplemento del Registro Oficial No. 463, 17 de noviembre 2004. Ley de Régimen Tributario Interno. [Internal Tax Regime Law]. Recuperado de https://n9.cl/da4j

Villagra-Cayamana, R. A., \& Zuzunaga-del-Pino, F. E. (2014). Tendencias del impuesto a la renta corporativo en Latinoamérica [Trends in corporate income tax in Latin America]. Derecho PUCP, (72), 163-202. 